\title{
Nach der Krebstherapie ist der Hausarzt gefragt
}

Forscher vom niederländischen Institut für Forschungen zum Gesundheitswesen (NIVEL) hatten in einer Studie die Zahl der Hausarztbesuche zwei bis fünf Jahre nach einer Krebsdiagnose - also mutmaßlich nach Abschluss der Tumortherapie - untersucht. Dafür werteten sie Angaben aus einer großen holländischen Datenbank zur Primärversorgung aus. 92 Praxen speisen hier zweimal jährlich Daten aus ihren elektronischen Patientenakten ein. In die Analyse flossen Angaben zu 1.256 Brustkrebspatientinnen, 503 Patienten mit Prostatakrebs und 487 Darmkrebspatienten ein. Als Kontrollen dienten für jeden Probanden aus der Tumorgruppe zwei nach Alter und Geschlecht gematchte Patienten ohne Krebsdiagnose, die den gleichen
Hausarzt aufsuchten. Wie sich zeigte, gingen Patienten nach einer Krebsdiagnose häufiger zum Hausarzt als die Kontrollpatienten. Die Kontaktzahlen steigerten sich um 24\% bei Brustkrebs-, $33 \%$ bei Prostatakrebs- und $15 \%$ bei Kolorektalkrebs-Patienten. Unterschiede zu den Kontrollprobanden ergaben sich vor allem bei jüngeren Patienten, die keine chronischen Erkrankungen aufwiesen.

Bereits in einer kürzlich veröffentlichten Studie waren die NIVEL-Wissenschaftler der Frage nachgegangen, aufgrund welcher gesundheitlichen Probleme Patienten, die eine Tumorerkrankung überstanden haben, häufiger zum Hausarzt gehen als ihre Vergleichspersonen [Heins MJ et al. Eur J Cancer. 2012 Aug 14. pii: S0959-8049(12)
00572-2]. Meist führten akute Beschwerden wie Infektionen, Bauchschmerzen oder Fatigue die Patienten zum Arzt. Dahinter könnten sich Nachwirkungen der Tumortherapie, aber auch vermehrte Sorgen um die Gesundheit verbergen.

„Angesichts des zu erwartenden Anstiegs der Zahl von Überlebenden einer Krebserkrankung sollten sich Hausärzte auf einen wachsenden Aufwand für die Nachbetreuung solcher Patienten einstellen", betonen die niederländischen Forscher. Multidisziplinäre Versorgungsstandards könnten hierbei hilfreich sein.

Robert Bublak

Heins M et al. Determinants of Increased Primary Health Care Use in Cancer Survivors. J Clin Oncol. 2012 Oct 15 [Epub ahead of print].

\section{kurz notiert}

\section{Forschungsstandort Deutschland}

Glioblastome differenzierbar +++

Ein internationales Team um Molekularbiologen des Deutschen Krebsforschungszentrums (DKFZ) konnte Glioblastome auf Basis molekularbiologischer Daten und klinischer Parameter in sechs Gruppen einteilen [Sturm D et al. Cancer Cell. 2012;22(4):425-37]. Bei Patienten aller Altersgruppen hatten sie Mutationen der Erbgutsequenz, das Aktivitätsmuster aller Gene, Erbgutverluste und Vervielfältigungen untersucht. Zudem wurde das Muster sogenannter Methylmarkierungen analysiert. Zusammen mit klinischen Daten führten diese Parameter zur Definition der sechs verschiedenen Glioblastiom-Gruppen. Die Einteilung ermöglicht es eventuell, differenziertere Therapien zu entwickeln und Studien gezielter zu planen.

Zugangskontrolle zu Erbinformationen +++ Erstmals wurde durch modernste DNASequenzierung die Position aller Nukleosomen im Erbgut embryonaler Stammzellen bestimmen [Teif VB et al. Nat Struct Mol Biol. 2012; 19(11): 1185-92]. Die Forscher, ebenfalls vom DKFZ Heidelberg,verglichen die NukleosomenAnordnung in embryonalen Stammzellen mit Zellen, aus denen sich Haut- und Gehirnzellen entwickeln. Es zeigte sich, dass sich die Anordnung völlig verändert. Der genaue Vorgang ist noch nicht bekannt, wird aber weiter beforscht um etwa besser zu verstehen, ob und wie unterschiedliche Krebsarten und Veränderungen der Nukleosomenorganisation assoziiert sind.

\section{Onkologen werden selten gefragt}

\section{Riesiger Infobedarf zu CAM bei Brustkrebs}

Anand von Fragebögen untersuchten Freiburger Wissenschaftler im Rahmen einer Querschnittstudie wie viele der teilnehmenden 170 Frauen mit Mammakarzinom aller Stadien Komplementäre und Alternative Medizin (CAM)-Methoden anwendeten, woher sie ihre Informationen bezogen und was sie sich wünschten.

Insgesamt nutzten $63 \%$ der Patientinnen CAM, in fortgeschrittenen Stadien sogar $80 \%$. Dabei handelte es sich vorwiegend um jüngere Frauen mit höherem Bildungsstand. So nahmen z.B. $66 \%$ von ihnen Vitamine und Mineralstoffe ein, $51 \%$ Mistelpräparate und $43 \%$ übten sich in Yoga und Entspannungstechniken. Als häufigste Gründe nannten die Frauen etwa, dass sie damit ihren Allgemeinzustand verbessern, ihre Selbstheilungskräfte wecken sowie aktiv etwas zu ihrer Gesundheit beitragen wollten.

Frauen ohne CAM-Erfahrung gaben an, dass sie von der Wirkung der konventionellen Therapiemaßnahmen größtenteils überzeugt seien (34\%). $31 \%$ von ihnen hatten noch nicht über die Nutzung weiterer Therapien nachgedacht.

Die Frauen sprachen das Thema CAM bei ihrem für die Standardversorgung zu- ständigen Onkologen eher nicht oder nur zögernd an. Jede Zweite verließ sich lieber auf Erfahrungen von Familienmitgliedern oder Freunden. 40\% fragten ihren Hausarzt nach Behandlungsmöglichkeiten und $39 \%$ suchten in verschiedenen Medien auch dem Internet - nach Informationen. Nur 20\% thematisierten CAM bei ihrem ambulanten Gynäkologen, beim Onkologen sogar nur $11 \%$.

Offenbar besteht aber ein dringender Wunsch der Patientinnen nach Wissen: $93 \%$ würden gerne bereits während der stationären onkologischen Therapie zu CAM-Möglichkeiten informiert werden. Als Grund für das ausbleibende Gespräch über CAM-Methoden gab jede vierte Patientin an, sie sei nicht danach gefragt worden, viele empfanden die Klinik auch als den falschen Ort für solche Themen.

Onkologen sollten laut den Autoren mehr auf ihre Patienten zugehen und von sich aus sachlich und informell über Begleittherapien sprechen. Christine Starostzik

Tautz E et al. Use of Complementary and Alternative Medicine in breast cancer patients and their experiences: A cross-sectional study. Eur J Cancer. 2012;48(17):3133-9. 